\title{
ARTICLE OPEN \\ Real-time optotracing of curli and cellulose in live Salmonella biofilms using luminescent oligothiophenes
}

\author{
Ferdinand X Choong ${ }^{1}$, Marcus Bäck ${ }^{2}$, Sara Fahlén ${ }^{1}$, Leif BG Johansson ${ }^{2}$, Keira Melican ${ }^{1}$, Mikael Rhen ${ }^{1,3}, \mathrm{~K}$ Peter R Nilsson ${ }^{2}$ and \\ Agneta Richter-Dahlfors ${ }^{1}$
}

Extracellular matrix (ECM) is the protein- and polysaccharide-rich backbone of bacterial biofilms that provides a defensive barrier in clinical, environmental and industrial settings. Understanding the dynamics of biofilm formation in native environments has been hindered by a lack of research tools. Here we report a method for simultaneous, real-time, in situ detection and differentiation of the Salmonella ECM components curli and cellulose, using non-toxic, luminescent conjugated oligothiophenes (LCOs). These flexible conjugated polymers emit a conformation-dependent fluorescence spectrum, which we use to kinetically define extracellular appearance of curli fibres and cellulose polysaccharides during bacterial growth. The scope of this technique is demonstrated by defining biofilm morphotypes of Salmonella enterica serovars Enteritidis and Typhimurium, and their isogenic mutants in liquid culture and on solid media, and by visualising the ECM components in native biofilms. Our reported use of LCOs across a number of platforms, including intracellular cellulose production in eukaryotic cells and in infected tissues, demonstrates the versatility of this optotracing technology, and its ability to redefine biofilm research.

npj Biofilms and Microbiomes (2016) 2, 16024; doi:10.1038/npjbiofilms.2016.24; published online 23 November 2016

\section{INTRODUCTION}

Biofilms are a natural multicellular form of bacterial life, which contribute to resistance against antibiotics, the host immune systems and environmental stresses. ${ }^{1}$ Biofilms enable bacteria to colonise abiotic surfaces (e.g., stainless steel, ${ }^{2}$ glass ${ }^{3,4}$ and plastics ${ }^{5}$ ) as well as biotic surfaces, such as epithelial cells and other tissue compartments. ${ }^{6,7}$ Embedded in an endogenously produced extracellular matrix (ECM), these mono- or poly-bacterial populations are difficult to eradicate. Although the overall dry mass of the biofilm may be substantial, microbial cells only constitute a small fraction, with the majority attributed to the extracellular polymeric matrix. ${ }^{8}$ The composition of ECM varies, but adhesins, amyloid-forming proteins and extracellular polysaccharides are ubiquitous ECM components. ${ }^{9}$ The amyloid curli fimbriae and bacterially produced cellulose have been identified as important ECM components for Escherichia coli, and Salmonella enterica serovars Enteritidis (S. Enteritidis) and Typhimurium (S. Typhimurium). ${ }^{10,11}$

Existing methods for biofilm detection and quantification are largely based on colorimetric assays using Crystal violet (CV), Congo red (CR) and Thioflavin derivatives. The CV assay, based on retention of molecules by hydrostatic interactions, provides only an indirect measure of biofilm, ${ }^{12}$ whereas the CR, Thioflavin and other hydrophobic molecules, which bind to ECM polysaccharides and amyloid proteins, enable direct quantification using fluorometric signals. ${ }^{13,14}$ The chemical nature of current dyes restricts their use to end-point measurements, with toxicity hindering their application in real-time studies of biofilm formation in vitro and in vivo.

In the research field of Alzheimer's disease, luminescent conjugated oligothiophenes (LCOs) have been used to detect amyloid protein aggregates both in vitro and in vivo. ${ }^{15-17}$
Surpassing the conventional amyloid ligands CR and Thioflavin, LCOs identify a broader subset of disease-associated protein aggregates and enable spectroscopic assignment of heterogeneous populations of deposits. ${ }^{18-21}$ The flexible conjugated thiophene backbone distorts in response to non-covalent electrostatic interactions with target molecules. This generates a conformation based, target specific, spectral signature, which in contrast to conventional fluorophores, exhibits an ON/OFF fluorescent signature. ${ }^{15-17}$ Interactions with amyloid proteins characteristically lead to the flattening of the molecular backbone and a more effective conjugation, causing a red-shift in the fluorescence excitation as well as increased fluorescence emission intensity. ${ }^{15-17}$ The excitation spectrum in particular is a direct reflection of the LCO backbone geometry.

Since amyloid proteins and fibrous polysaccharides are major ECM constituents, we hypothesised a novel use of LCOs as non-bactericidal, conformational sensitive fluorescent probes for real-time detection and differentiation of these essential components of Salmonella biofilms, a method we define as optotracing. To our knowledge, no conventional dyes, or other available techniques are able to monitor dynamic biofilm formation and concurrently differentiate between curli fibres and cellulose under live conditions. Visualising the dynamics of biofilm formation under live conditions at a resolution where individual biofilm components are detected is thus hampered. To address this need, we employed two prototype, non-toxic LCO molecules to dynamically detect and differentiate between curli fibres and cellulose polysaccharides in S. Enteritidis and $S$. Typhimurium forming biofilm on abiotic surfaces, agar plates, in liquid cultures, intracellularly in eukaryotic cells, and in mouse liver.

\footnotetext{
${ }^{1}$ Swedish Medical Nanoscience Center, Department of Neuroscience, Karolinska Institutet, Stockholm, Sweden; ${ }^{2}$ Division of Chemistry, Department of Physics, Chemistry and Biology, Linköping University, Linköping, Sweden and ${ }^{3}$ Department of Microbiology, Tumor and Cell biology, Karolinska Institutet, Stockholm, Sweden.

Correspondence: A Richter-Dahlfors (Agneta.richter.dahlfors@ki.se)

Received 19 May 2016; revised 15 July 2016; accepted 8 September 2016
} 


\section{RESULTS}

Fluorescent differentiation of ECM components using luminescent oligothiophenes

Two LCOs, h-HTAA and h-FTAA (Figure 1a), selected from our library of synthesised LCO molecules for their amyloid sensitivity, were screened for their suitability as optotracers of biofilm ECM components on an isogenic collection of $S$. Enteritidis based on the wild-type (wt) strain 3934 (Supplementary Table 1). To facilitate analysis of surface-bound biofilm formed at the air-liquid interface, bacteria were grown in wells with inclined square glass coverslips (Figure 1b). After gentle removal of the coverslips, LCOs were first applied directly onto the surfaces, which then were prepared for microscopic analysis. Fluorescence microscopy of the biofilms demonstrated distinct labelling, suggesting that h-HTAA (green) and h-FTAA (red) fluorescence signals can complement phase contrast when visualising biofilm morphology (Figure 1c and Supplementary Figure 1). In contrast, no fluorescent signals were identified from a $\Delta \operatorname{csg} D$ mutant strain unable to produce curli and cellulose (Figure 1d). Individual contribution of the two ECM elements to the positive LCO-biofilm staining was analysed using $\triangle b c s A$ (curli $^{+}$cellulose $^{-}$) and $\triangle \operatorname{csg} A$ (curli $^{-}$cellulose ${ }^{+}$) mutant strains. Phase contrast microscopy of the cellulose-deficient mutant (strain $\triangle b c s A$ ) showed similar morphology to the $w t$, and distinct fluorescence signals from both LCOs (Figure 1e). A thin and brittle bacterial layer, typically formed in the absence of curli, ${ }^{22}$ was observed in the $\Delta \operatorname{csg} A$ mutant strain and LCO staining revealed distinct fluorescence that was more pronounced in areas with higher cell density (Figure 1f).

Growth curves of the wt strain in the absence and presence of LCOs revealed a common generation time of $23 \pm 1 \mathrm{~min}$, indicating that neither h-HTAA nor h-FTAA exert bacteriostatic or bactericidal effects (Figure 2a). This corroborated previous demonstrations of a non-toxic nature of the oligothiophene family on eukaryotic cells in vitro and in intravital mouse models. ${ }^{23,24}$ We therefore tested LCOs in live cultures, to monitor the extracellular appearance of curli fibres and cellulose during growth. A custom-designed, small-volume 96-well assay (Supplementary Figure 2) enabled analysis of pellicle and surface-attached biofilm at selected time points using spectrophotometric recordings in a standard plate reader. Fluorescence analysis of wt cultures in the presence of h-HTAA or h-FTAA (Figure 2b) showed a comparable increase of biofilm growth to parallel CV assays (Figure 2c). We next addressed if LCOs can a

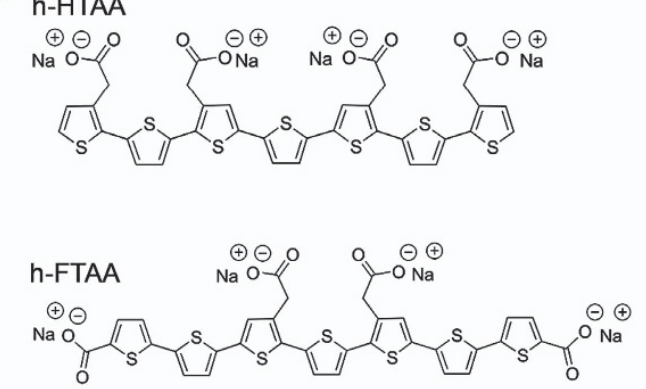

C

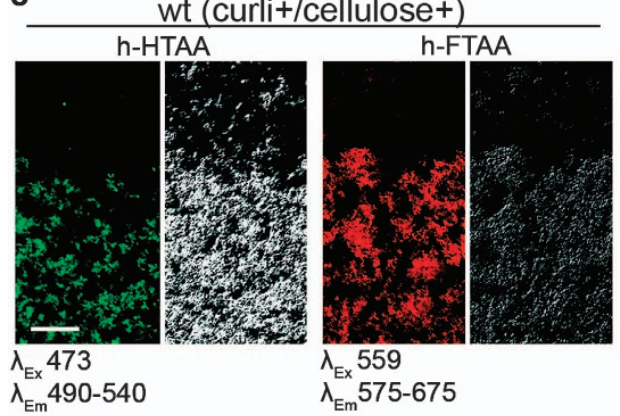

e

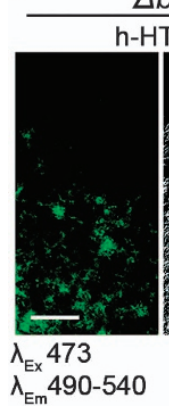

$\triangle b c s A$ (curli+/cellulose-)
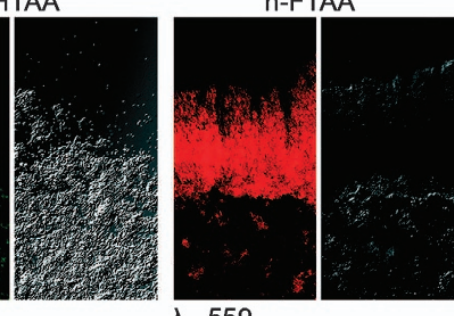

$\lambda_{\mathrm{Ex}} 559$

$\lambda_{\mathrm{Em}}^{\mathrm{Ex}} 575-675$ b

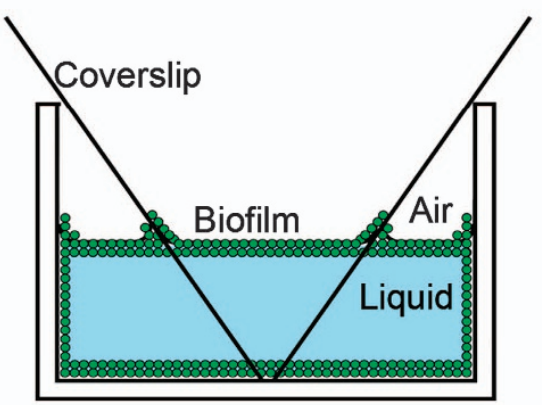

d

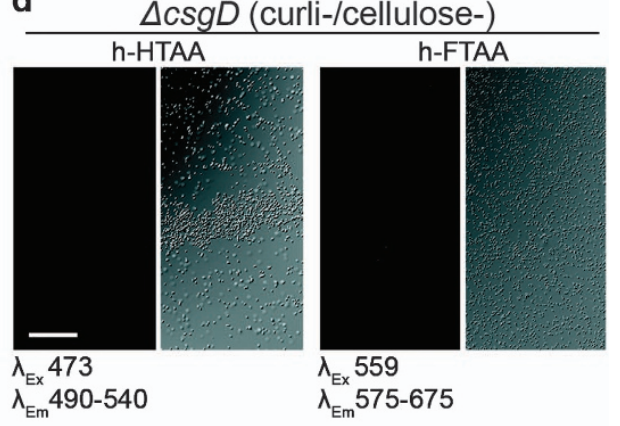

f

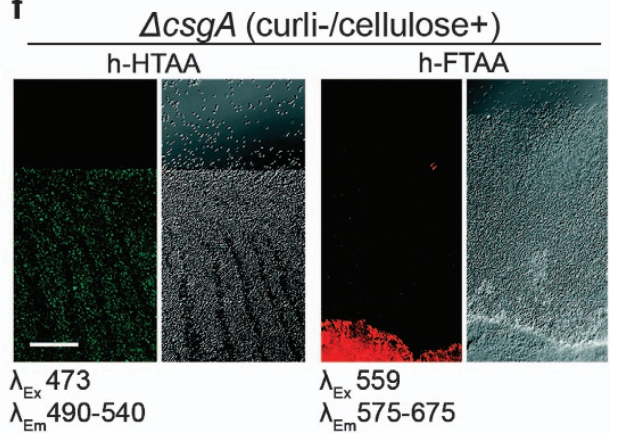

Figure 1. LCO staining patterns distinguish Salmonella biofilms. (a) Structure of h-HTAA and h-FTAA. (b) Schematic of the incline glass coverslip setup enabling microscopic analysis of biofilm at air-liquid interface after removal of coverslips. (c-f) Fluorescence confocal microscopy using indicated excitation and emission wavelengths (left) and transmission confocal microscopy (right) of h-HTAA- and h-FTAA-stained biofilms from strains 3934 (c) wt, (d) $\Delta \operatorname{csg} D,(\mathbf{e}) \Delta b \operatorname{cs} A$ and (f) $\Delta \operatorname{csg} A$ with indicated curli and cellulose phenotypes. Single optical sections are shown. Scale bar $=50 \mu \mathrm{m}$. 
a

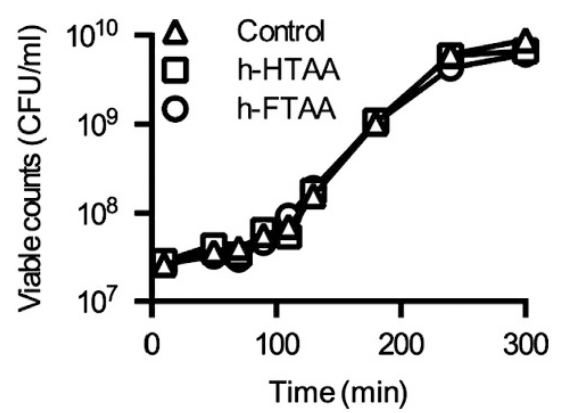

d

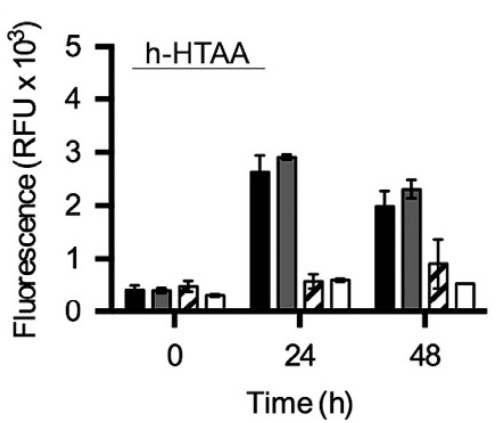

b
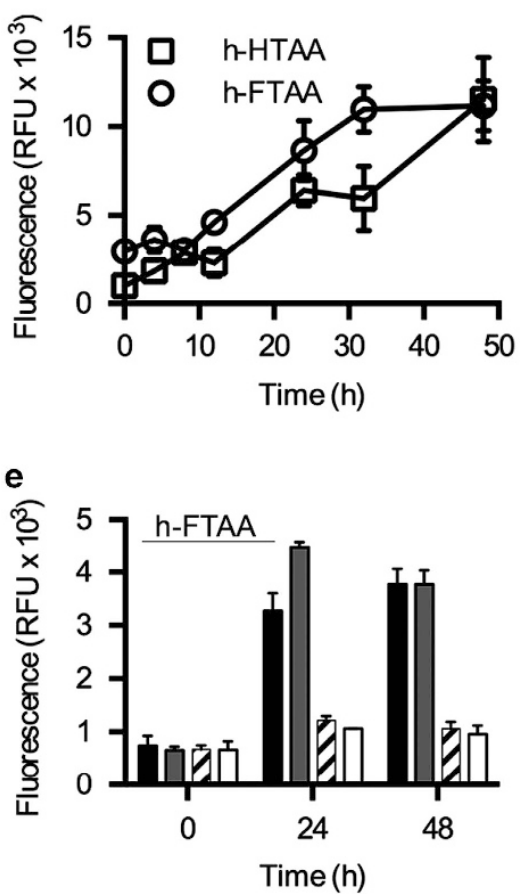

C

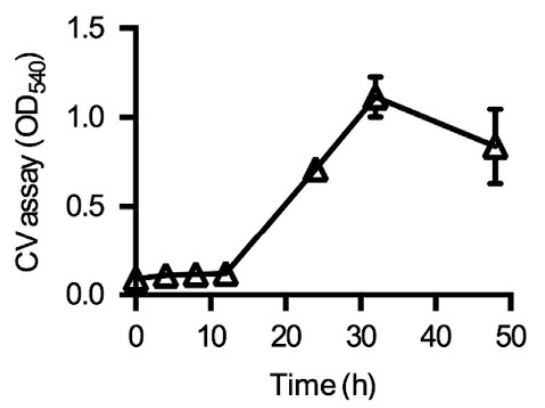

f

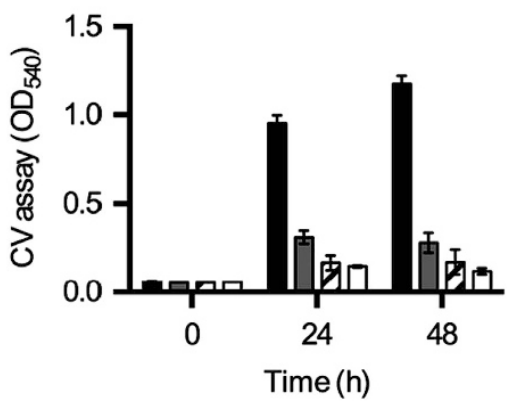

Figure 2. LCO-based fluorometric biofilm quantification in a small-volume 96-well assay. (a) Growth curve, shown as viable counts, of strain 3934 wt cultured in the absence (control) and presence of h-HTAA and h-FTAA. (b and $\mathbf{c}$ ) End point quantification of biofilm formed by 3934 wt at indicated times based on (b) fluorescence from cultures grown in the presence of h-HTAA and h-FTAA and (c) the crystal violet assay. (d-f) Quantification of biofilm formed at 24 and $48 \mathrm{~h}$ by $3934 \mathrm{wt}(\square), \Delta b \operatorname{cs} A(\square), \Delta \operatorname{csg} A$ (ש/A) and $\Delta \operatorname{csg} D$ ( $\square$ ) based on fluorescence from (d) h-HTAA, (e) h-FTAA, and based on the (f) crystal violet assay. Data represent $n: 1$ of 3 with standard deviations shown. CFU, colony forming units; CV, crystal violet; RFU, relative fluorescence units.

distinguish between different biofilm phenotypes by analysing wt and mutant biofilm formation in the small-volume 96-well assay. Fluorometric read-outs (excitation wavelength $\left(\lambda_{\mathrm{Ex}}\right)$ of $405 \mathrm{~nm}$ and emission wavelength $\left(\lambda_{\mathrm{Em}}\right)$ of $556 \mathrm{~nm}$ ) taken at set time points from a continuous culture grown in the presence of h-HTAA or h-FTAA, revealed that the wt and $\triangle b c s A$ (curli $^{+}$cellulose $^{-}$) strains produced high quantities of biofilm (Figure $2 \mathrm{~d}, \mathrm{e}$ ). In contrast, $\triangle \operatorname{csg} A$ (curli $^{-}$cellulose ${ }^{+}$) and $\Delta \operatorname{csg} D$ (curli ${ }^{-}$cellulose ${ }^{-}$) mutants produced only low amounts. These results supported the microscopy-based demonstration of the $\triangle b c s A$ mutant as a substantial biofilm producer (Figure 1e). This result differed however from traditional CV assay results, which showed poor biofilm formation by all three mutants (Figure 2f). LCO-based detection appears to differentiate between biofilm phenotypes, with substantially improved sensitivity in biofilms with curli as the major ECM component.

\section{Non-disruptive analysis of biofilm formation}

When studying the kinetics of biofilm formation, longitudinal experiments should ideally be performed with minimum disturbance, such as washing procedures. To test whether LCOs can be used for non-disruptive studies, strains were cultured in h-HTAA-supplemented medium in 96-well plates, and the fluorescence of each well was directly recorded at 0,24 and $48 \mathrm{~h}$. A 4-6-fold fluorescence increase was observed across all strains (Figure 3a) compared with equivalently washed, end-point experiments (Figure 2d). The signals, however, no longer distinguished between the biofilm phenotypes, suggesting that the complex composition of the LB culture contributes to a high background, emitting in the same wavelength window as h-HTAA. To test this hypothesis, excitation spectra from each $24 \mathrm{~h}$ culture were collected. Identical spectra, sharing one characteristic peak, were observed for all strains irrespective of the amounts and phenotypes of the biofilms (Figure 3b). This suggested that h-HTAA binds a biofilm component different from curli and cellulose, which is ubiquitously present in all cultures. While limited in use for the purpose of the present study, h-HTAA still represents an interesting alternative to current biofilm dyes as a general non-bacteriocidal, fluorescence alternative for endpoint studies.

The h-FTAA was, however, able to differentiate biofilm phenotypes in the longitudinal assays (Figure 3c). With h-FTAA, the cellulose-deficient mutant $\triangle b c s A$ showed significant amounts of biofilm, with a 4-5 fold increase in signal intensity at $24 \mathrm{~h}$, a similar pattern to the washed endpoint experiments (Figure 2e). No further increase was observed, indicating that the bulk of this curli-based biofilm had formed in the first $24 \mathrm{~h}$. Despite high background, spectral analysis showed increased fluorescence intensity from curli-producing wt and $\Delta b c s A$ compared with the curli-deficient strains at $\lambda_{\mathrm{Ex}} 405 \mathrm{~nm}$, which may represent a signal unique to h-FTAA bound to curli fibres (Figure $3 \mathrm{~d}$ ). A unique excitation peak also appeared at $\sim 480 \mathrm{~nm}$ in wt and $\triangle \operatorname{csgA}\left(\right.$ curli $^{-}$cellulose $^{+}$) strains suggesting the simultaneous detection of a second target.

Based on the genetic make-up of our Salmonella strains, cellulose, whose biosynthesis is a shared trait in the wt and $\Delta \operatorname{csg} A$ (curli $^{-}$cellulose ${ }^{+}$) strains appears to be a strong candidate to serve as a second binding target for h-FTAA. To test this hypothesis, the excitation spectra of h-FTAA combined with different concentrations of pure cellulose was obtained. The spectra showed an evident peak with $\lambda_{\max }$ at $\sim 480 \mathrm{~nm}$ when emission was recorded at $545 \mathrm{~nm}$ (Figure $3 \mathrm{e}(5 \mathrm{mg} / \mathrm{ml}$ ) and Supplementary Figure 3 $(0.04-2.5 \mathrm{mg} / \mathrm{ml})$. This peak is comparable to that generated by the cellulose-producing Salmonella strains (Figure $3 \mathrm{~d}$ ), and demonstrates the exclusive spectral signature of h-FTAA binding to cellulose. This finding extends the applicability of LCOs from conformation-sensitive spectral probes detecting amyloid protein aggregates ${ }^{15-17}$ to also include polysaccharides. 

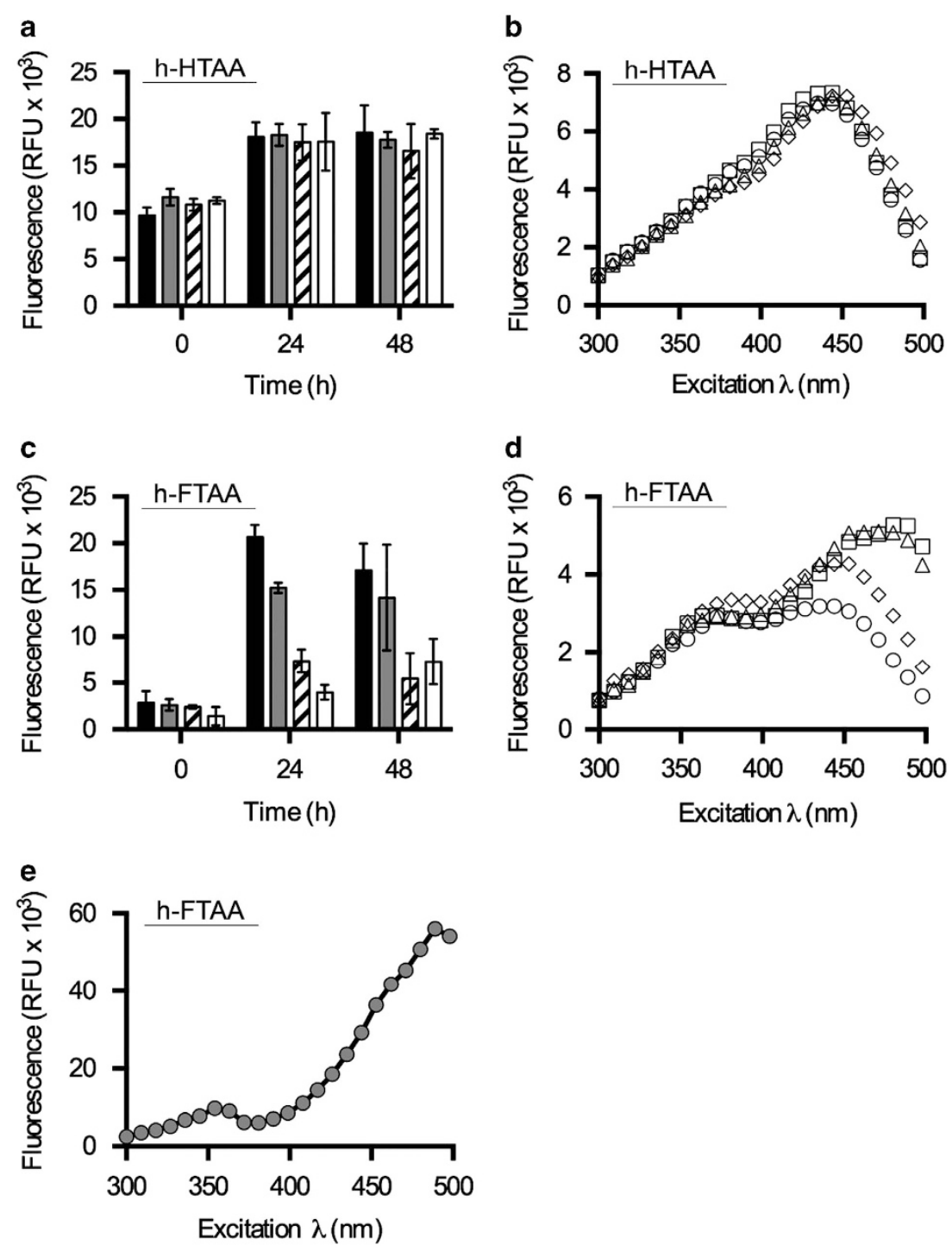

Figure 3. Individual and simultaneous LCO-based quantification of curli and cellulose in longitudinal biofilm cultures. (a) Fluorescence of h-HTAA at 24 and $48 \mathrm{~h}$ in non-disrupted liquid cultures of 3934 wt ( $\square$ ), $\Delta b \operatorname{cs} A(\square), \Delta \operatorname{csg} A$ (四) and $\Delta \operatorname{csg} D$ ( $\square$ ). (b) Spectra of h-HTAA in cultures at $24 \mathrm{~h}$ of $3934 \mathrm{wt}(\triangle) \triangle b \operatorname{cs} A(\diamond), \Delta \operatorname{csg} A(\square)$ and $\triangle \operatorname{csg} D(0)$, with emission read at $545 \mathrm{~nm}$. (c) Fluorescence of h-FTAA at 24 and $48 \mathrm{~h}$ in non-disrupted liquid cultures of 3934 wt $(\square), \Delta b \operatorname{cs} A(\square), \Delta \operatorname{csg} A$ (四) and $\Delta \operatorname{csg} D(\square)$. (d) Spectra of h-FTAA at $24 \mathrm{~h}$ of 3934 wt $(\triangle) \Delta b \operatorname{cs} A(\diamond)$ ), $\Delta \operatorname{csg} A(\square)$ and $\Delta \operatorname{csg} D(0)$, with emission read at $545 \mathrm{~nm}$. (e) Spectra of h-FTAA mixed with cellulose $(5 \mathrm{mg} / \mathrm{ml})$ with emission read at $545 \mathrm{~nm}$. Data represent $n$ : 1 of 3 with standard deviations shown in a and c. RFU, relative fluorescence units.

\section{Spectral morphotyping of biofilm}

More than $90 \%$ of S. Typhimurium and S. Enteritidis strains produce a characteristic red, dry and rough colony morphology on solid media, the so-called rdar morphotype. ${ }^{25}$ This threedimensional architecture is formed by highly ordered spatial arrangement of cellulose filaments and curli fibre networks. ${ }^{26}$ We analysed whether LCOs can be used for definitive spectral morphotyping of bacterial colonies. Having ascertained that the strains showed the expected rdar morphotypes on CR plates ${ }^{27}$ (Figure 4a), we added h-FTAA to re-suspensions of 3-day-old colonies grown on LB agar plates without salt, and performed spectral analysis. Comparison of differently sized colonies at different developmental stages was enabled by normalising each spectrum such that each data point is represented as a percentage of the largest emitted fluorescence of the excitation spectra. A distinct red-shifted cellulose-specific peak was observed at $480 \mathrm{~nm}$ in wt and $\triangle \operatorname{csg} A$ (curli $^{-}$cellulose $^{+}$) colonies, overlapping the signal from h-FTAA binding pure cellulose (Figure $4 b$ ).
The peak was absent in $\triangle c s g D$ and $\triangle b c s A$ colonies, both genetically incapable of cellulose production.

Rdar morphotyping on $\mathrm{CR}$ plates requires that a bacterial colony, often initiated from a $25 \mu \mathrm{l}$ drop of $\sim 10^{5}$ c.f.u., reach a sufficient size for visual inspection, which usually takes 3 days (Figure 4c). To enable non-biased profiling during biofilm development on LB plates without salt, we analysed daily harvests of individual wt colonies, originating from a single bacterium, for the presence of cellulose. Spectra of colony re-suspensions stained with h-FTAA consistently showed a cellulose peak at $480 \mathrm{~nm}$ on day 2 , and persisting on day 3 (Figure $4 d$ ). The presence of this peak was less consistent on day 1 (Figure 4e). The variability at this early stage reflects a changing ratio of unbound and bound h-FTAA as the amount of cellulose in the colony increases during biofilm maturation. In some day 1 colonies, cellulose was produced which bound all h-FTAA molecules, thus generating the $480 \mathrm{~nm}$ peak (Figure 4e, long arrow). In colonies with less cellulose, the higher proportion of unbound h-FTAA was observed 
a
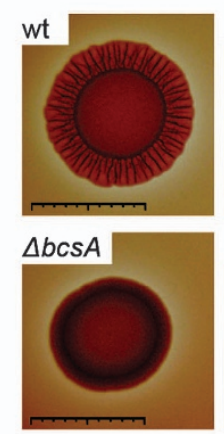

c

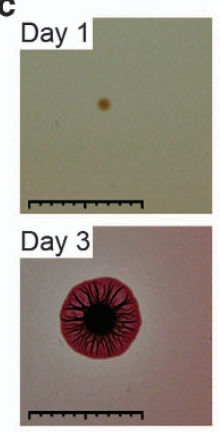

b
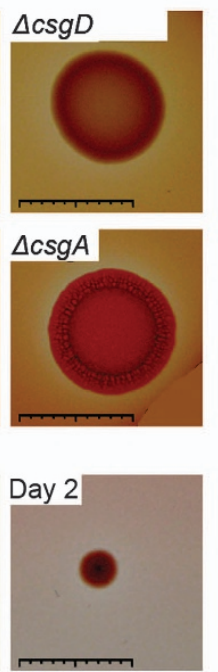

d
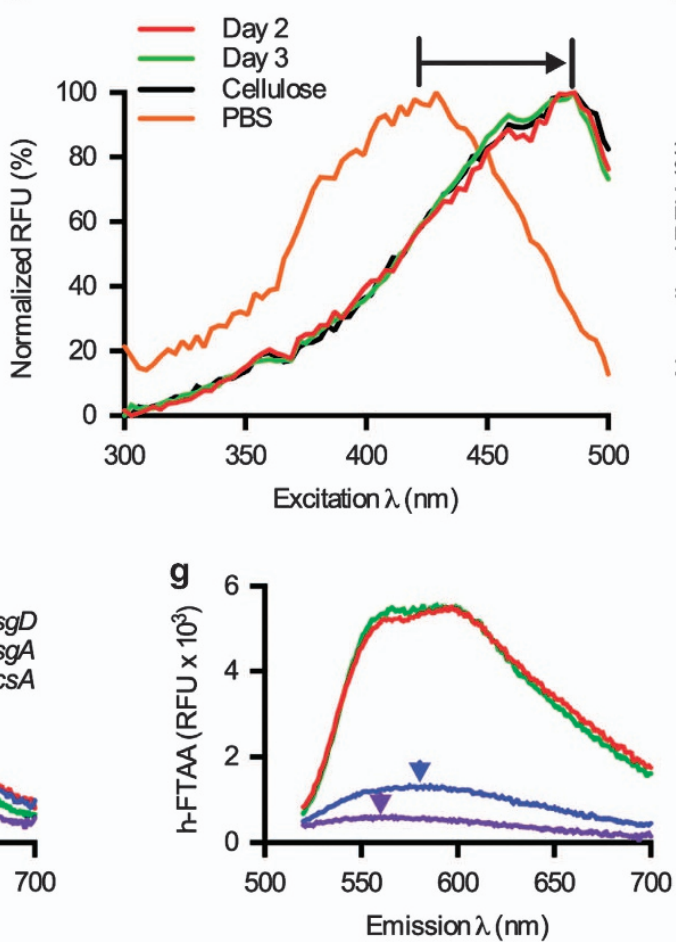

e
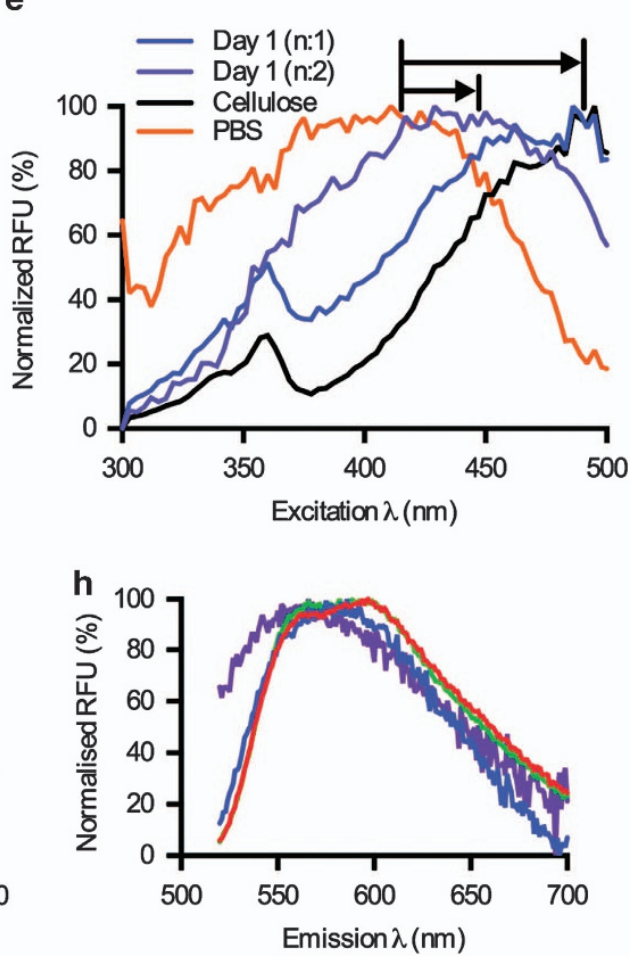

Figure 4. LCO-based morphotyping of Salmonella biofilms from agar plates. (a) Morphotypes of strain $3934 \mathrm{wt}, \Delta \operatorname{csg} D, \Delta b \operatorname{cs} A$ and $\Delta \operatorname{csg} A$ based on the drop assay on Congo red plates. (b) Normalised spectra of h-FTAA mixed with re-suspended biofilm colonies harvested from indicated strains grown for $48 \mathrm{~h}$ on LB agar w/o salt, with emission read at $545 \mathrm{~nm}$. h-FTAA mixed with cellulose and PBS were assayed in parallel for reference. (c) Morphotype of a $3934 \mathrm{wt}$ biofilm colony originating from an individual bacterium on Congo red plates monitored for three consecutive days. (d and e) Spectra of h-FTAA mixed with harvested $3934 \mathrm{wt}$ biofilm colonies at (d) days 2 and 3, and (e) day 1, including cellulose and PBS for reference. Arrows indicate the shift in $\lambda_{\max }$ for h-FTAA in the presence of various amounts of cellulose. $n: 1$ of 5 in $\mathbf{b}$ and $\mathbf{d}$, $n: 2$ of 5 in e. Scale bars $=1 \mathrm{~cm}$. (f) Emission spectra of h-FTAA-supplemented cultures of strain $3934 \mathrm{wt}, \Delta b \operatorname{cs} A, \Delta \operatorname{csg} A$ and $\Delta \operatorname{csg} D$ after $24 \mathrm{~h}$ incubation, using excitation at $405 \mathrm{~nm}$ for curli detection. (g) Same experimental setup as in f using excitation at $500 \mathrm{~nm}$ for cellulose detection. Arrows indicate $\lambda_{\max }$ of emission in $\Delta \operatorname{csg} A$ and $\Delta \operatorname{csg} D$ mutant strains. (h) Normalised fluorescence spectra for cellulose detection from g. Data represent $n$ : 1 of 3 . RFU, relative fluorescence units.

as an intermediate degree of red-shifted $\lambda_{\max }$ (Figure $4 \mathrm{e}$, short arrow). The transition of $\lambda_{\max }$ reflects cellulose production during the transition between different bacterial growth stages of colonies growing on solid medium. Such transient events are invisible to eye inspection (Figure 4c).

Defining optical settings for simultaneous, dual detection of cellulose and curli

The binding of h-FTAA to amyloid curli protein and cellulose suggests its possible application as an optotracer for simultaneous, dual detection of ECM components. We applied the small-volume 96-well assay of defined bacterial cultures to test whether we could identify discrete spectral signatures for each target. To identify the optimum emission wavelength $\left(\lambda_{\mathrm{Em}}\right)$ of
h-FTAA bound to curli, we collected the emission spectra with excitation at $405 \mathrm{~nm}$. The three biofilm-forming strains produced red-shifted emission peaks at $\lambda_{\max } 550-560 \mathrm{~nm}$, compared with a $\lambda_{\max } 525 \mathrm{~nm}$ in the non-biofilm forming $\Delta \operatorname{csg} D$ mutant (Figure $4 \mathrm{f}$ ). Increased fluorescence amplitude was only observed in curlicontaining biofilms (wt and $\triangle b c s A$ ), corroborating the finding in Figure 3c. This suggests $\lambda_{\mathrm{Em}} 550-560 \mathrm{~nm}$ as an optimum range for curli detection. Cellulose does not appear to contribute to the background signal, since the red-shift appearing in the cellulose-expressing $\Delta \operatorname{csg} A$ curli mutant was not associated with a fluorescence intensity increase.

To define the optimal emission wavelength for cellulose detection, excitation at $500 \mathrm{~nm}$ was used, as this wavelength was observed to maximise the signal-to-background ratio 
(Figure 3d). Two prominent emission peaks at $\sim 560 \mathrm{~nm}$ and $\sim 597 \mathrm{~nm}$ were seen for $\mathrm{h}-\mathrm{FTAA}$ binding to the cellulose-containing biofilm from wt and $\triangle \operatorname{csg} A$ (Figure $4 \mathrm{~g}$ ). Strains lacking this ECM component $(\triangle b c s A$ and $\triangle c s g D)$ showed h-FTAA binding to curli or an unknown component, inferred from the weak $\lambda_{\max }$ signals at 566 and $586 \mathrm{~nm}$ that were only noticeable following data normalisation (Figure 4h). Detection above $597 \mathrm{~nm}$ in celluloseproducing strains resulted in less background than detection at $560 \mathrm{~nm}$. Ensuring minimal contribution of background, optimal spectral parameters for h-FTAA based cellulose detection were identified as $\lambda_{\mathrm{Ex}} 500 \mathrm{~nm}$ and $\lambda_{\mathrm{Em}} 600 \mathrm{~nm}$. Combining this with spectral parameters optimal for curli $\left(\lambda_{\mathrm{Ex}} 405 \mathrm{~nm}, \lambda_{\mathrm{Em}} 556 \mathrm{~nm}\right)$ thus enables dual detection of the ECM components.

Real-time detection of curli and cellulose in liquid bacterial cultures

In contrast to traditional fluorophores, fluorescence from the LCOs is modulated by the molecular geometry. As the geometry changes in the bound versus unbound state, fluorescence intensity increases at a given wavelength, showing an ON-like switch as the corresponding binding target appears. We evaluated LCOs as dynamic optotracers of curli and cellulose during biofilm formation, using spectrophotometric recordings of h-FTAA added to bacterial cultures in the small-volume 96-well format. Bacterial GFP expression (plasmid p2777-Supplementary Table 1) enabled simultaneous recording of bacterial growth based on GFP expression ( $\left.\lambda_{\mathrm{Ex}} 445 \mathrm{~nm}, \lambda_{\mathrm{Em}} 510\right)$, and h-FTAA detection of curli $\left(\lambda_{\mathrm{Ex}} 405 \mathrm{~nm}, \lambda_{\mathrm{Em}} 556 \mathrm{~nm}\right)$ and cellulose $\left(\lambda_{\mathrm{Ex}} 500 \mathrm{~nm}, \lambda_{\mathrm{Em}} 600 \mathrm{~nm}\right)$. The appearance of the curli and cellulose spectral signatures coincided with the shift from late logarithmic to early stationary phase at circa $15 \mathrm{~h}$ in the wt culture (Figure 5a). Significant signal increase demonstrated pronounced secretion and assembly of both ECM components during early stationary phase. After $\sim 5 \mathrm{~h}$, production ceased and the signals remained at a consistent level throughout late stationary phase. No signal was detected in the curli- and cellulose-deficient strain $\triangle \operatorname{csg} D$-p2777 (Figure 5b).

In the curli-deficient $\Delta \operatorname{csg} A-p 2777$ strain, cellulose was expressed $4 \mathrm{~h}$ earlier than the wt culture, during the exponential
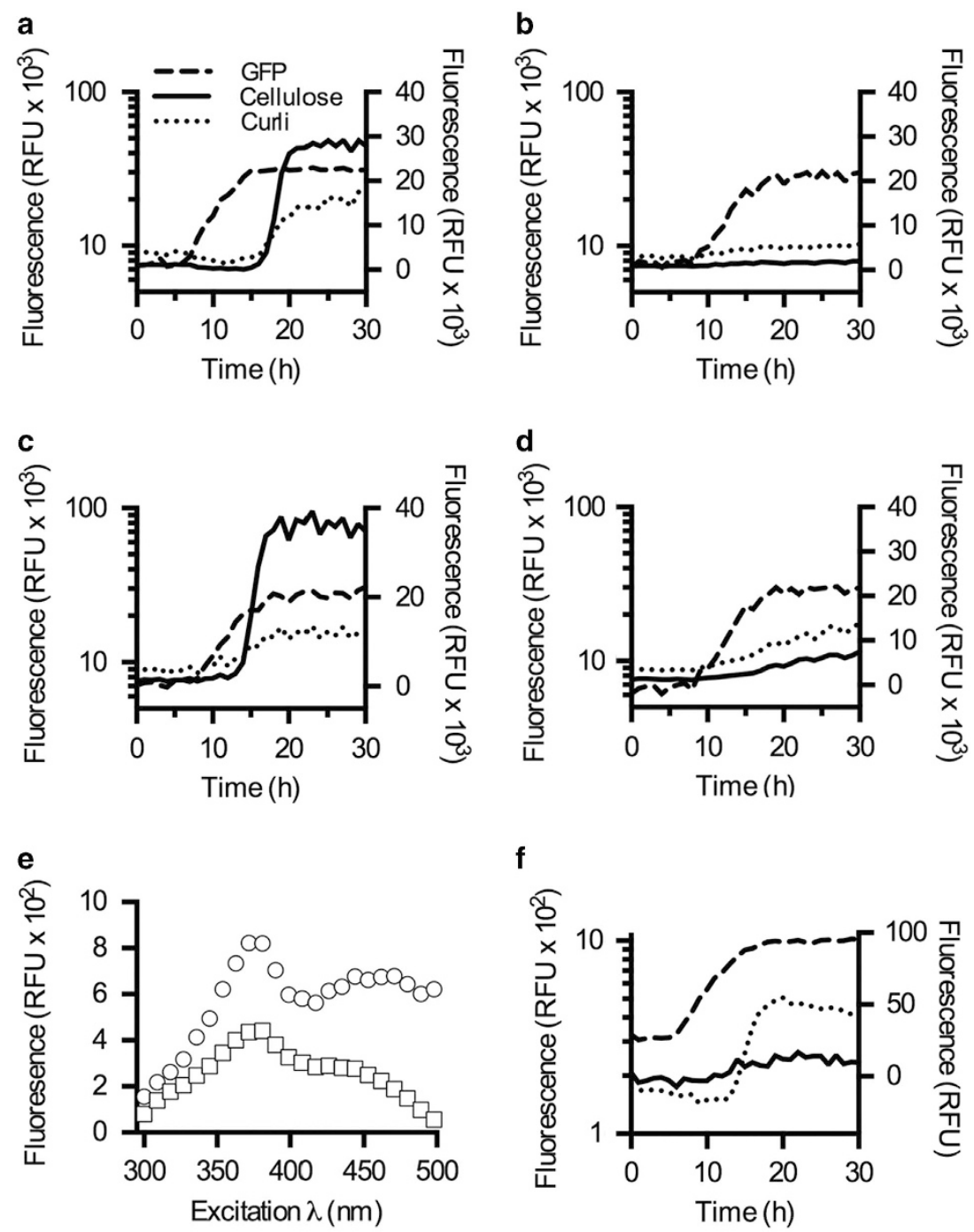

Figure 5. h-FTAA enables simultaneous, real-time detection of curli and cellulose in liquid Salmonella cultures. (a-d) Combined real-time recording of bacterial growth (left $y$-axis) measured by intensity of GFP ( $\lambda_{\mathrm{Ex}} 445 \mathrm{~nm}, \lambda_{\mathrm{Em}} 510 \mathrm{~nm}$ ), and h-FTAA staining extracellular curli $\left(\lambda_{\mathrm{Ex}} 405 \mathrm{~nm}, \lambda_{\mathrm{Em}} 556 \mathrm{~nm}\right)$ and cellulose $\left(\lambda_{\mathrm{Ex}} 500 \mathrm{~nm}, \lambda_{\mathrm{Em}} 600 \mathrm{~nm}\right.$; right $y$-axis) in liquid cultures of strains 3934 (a) wt, (b) $\Delta c s g D$, (c) $\Delta c s g A$ and (d) $\triangle b c s A$ harbouring plasmid p2777. (e) Autofluorescence from LB medium only ( $\square$ ), as well as the combined background fluorescence from biofilm-forming, GFP-expressing bacteria in LB medium (O). (f) Combined real-time recording of bacterial growth, monitored by GFP expression (dashed line, left $y$-axis) from strain 3934 wt p2777, and appearance of cellulose detected by h-FTAA (right $y$-axis) in the absence (dotted line) and presence (solid line) of the cellulose-digesting enzyme cellulase. 
a

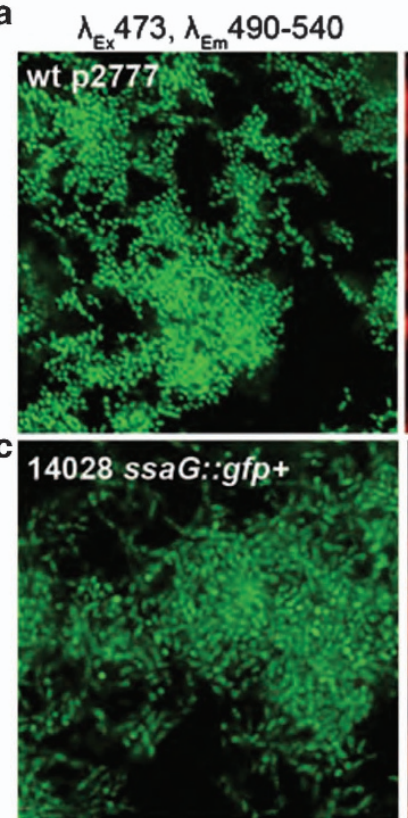

d

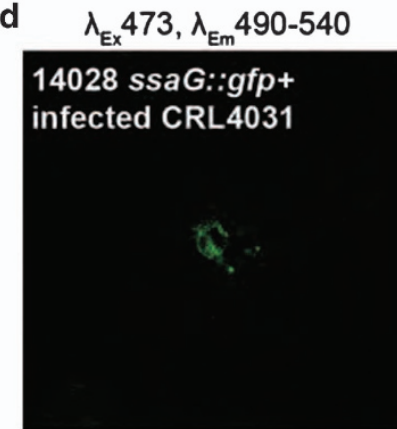

e 14028 ssaG::gfp+

infected RAW264.7

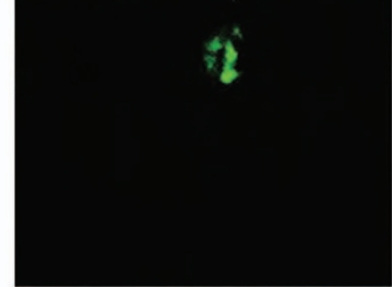

14028 ssaG::gfp+ infected liver
$\lambda_{E x} 559, \lambda_{E m} 575-675$
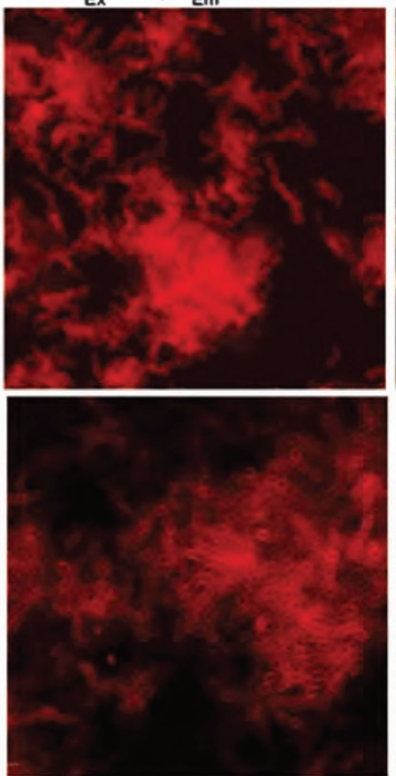

$\lambda_{E x} 473, \lambda_{E m} 590-655$
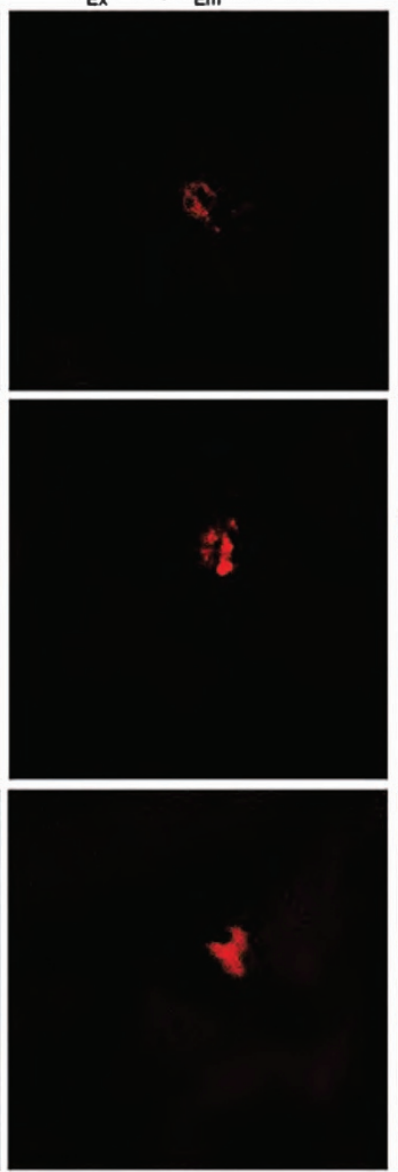

b

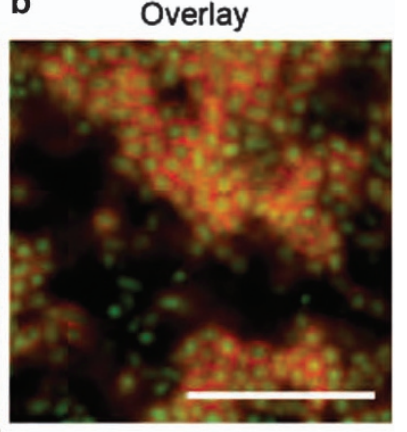

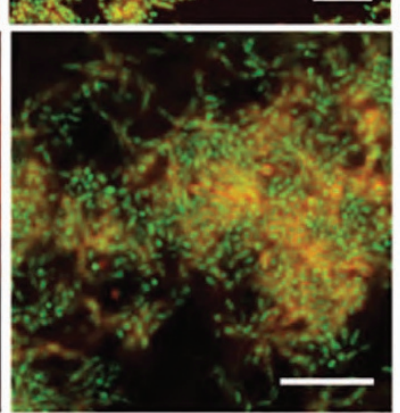

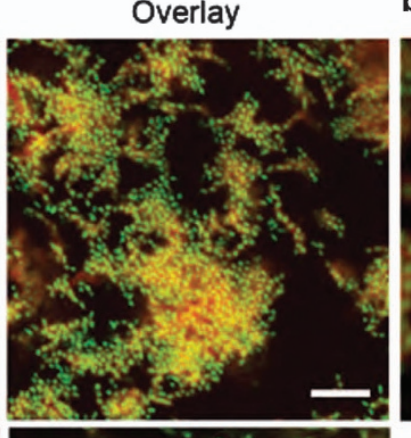

Overlay + Hoechst 33342
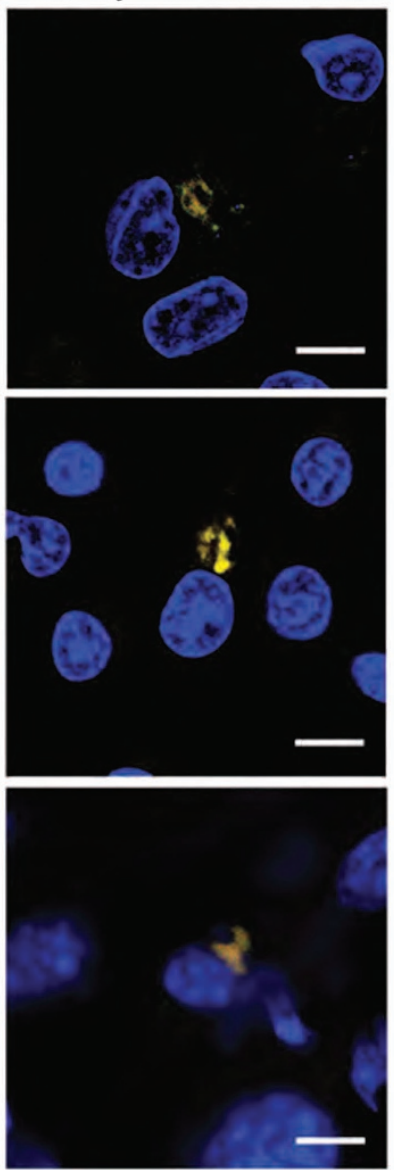

Figure 6. Visualisation of ECM components in biofilms formed by $S$. Enteritidis and S. Typhimurium in the presence of h-FTAA. (a-c) Fluorescence confocal microscopy of unfixed biofilm formed by S. Enteritidis strain 3934 wt p2777 at (a) lower and (b) higher

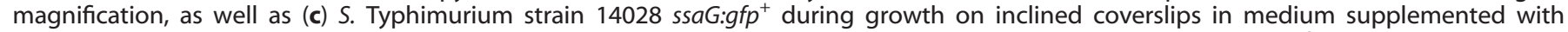
h-FTAA. Bacteria (green) and ECM (red) are detected at indicated wavelengths, representing the microscopes' pre-defined detection settings for GFP and Cy3. (d-f) Fluorescence confocal microscopy images of 14028 ssaG:gfp ${ }^{+}$(green) infected (d) CRL-4031 epithelial cells, and (e) RAW264.7 macrophage cell and in (f) sections of mouse livers stained with h-FTAA (red). Staining with Hoechst 33324 shows nuclei (blue) of each cell type. Single optical sections are shown. Scale bar $=10 \mu \mathrm{m}$. 
growth phase (Figure $5 c$ ). Multiple comparison analysis (ANOVA) revealed that this difference was statistically significant (Supplementary Figure 4 and Supplementary Table 2). The sharp increase leveled off, and remained constant from early stationary phase onwards. In the curli detection window, a small non-specific signal appeared, which we could ascribe to the broad emission range of h-FTAA bound to cellulose (Figure $4 \mathrm{~g}$ ).

The cellulose-deficient strain $\triangle b c s A-p 2777$, showed a slight signal in the cellulose detection window (Figure $5 \mathrm{~d}$ ), which was identified as GFP 'bleed-through' by an excitation scan of a planktonic GFP-producing culture (Figure 5e). Curli production increased only gradually throughout the time course of an experiment, a trend differing from the conventional sigmoidal dynamics of ECM formation (Figure 5a). These data suggest that the absence of cellulose de-regulates the production of curli fibres. The kinetics of immediate LCO fluorescence upon amyloid assembly ${ }^{28}$ implies that the gradual signal increase directly reflects the appearance of curli in the biofilm. The topic of cellulose- and curli co-regulation in biofilm needs further study, studies in which LCOs can play a major role.

To verify the specificity of h-FTAA as a tool for real-time in situ measurements of assembled biofilm components, we grew the wtp2777 strain in the presence a cellulose-degrading enzyme. Whereas increased GFP fluorescence indicated bacterial growth was not affected by the addition of cellulase, the h-FTAA signal was suppressed due to the effective elimination of the cellulose polysaccharides (Figure 5f). Taken together, our data show h-FTAA to be able to specifically detect the expression of cellulose and curli simultaneously in a continuously growing culture, opening up many exciting possibilities for further kinetic studies.

\section{Fluorescence imaging of biofilm ECM}

Fluorescence-based microscopy techniques are fundamental tools for the better understanding of biological events. As a lab with a strong interest in microscopy, we analysed the use of LCOs for in situ staining of ECM in growing biofilms. Using the inclined coverslip set-up, cultures of wt-p2777 in medium supplemented with h-FTAA-enabled incorporation of the optotracer molecules into biofilm formed at the air-liquid interface of the coverslips. Without any additional treatment, confocal microscopy was performed directly on coverslips removed at $24 \mathrm{~h}$. Large communities of distinct rod-shaped GFP-expressing bacteria were observed, surrounded by dense mesh-like structures visualised by fluorescence from h-FTAA bound to the ECM (Figure $6 a$ and Supplementary Figure $5 a, b)$. The fluorescence of h-FTAA was detectable using standard microscopy settings for Cy3 $\left(\lambda_{\mathrm{Ex}} 559 \mathrm{~nm}, \lambda_{\mathrm{Em}} 575-675 \mathrm{~nm}\right)$. Higher magnification revealed the spatial relationship more clearly, showing red ECM filling the extracellular spaces between green bacterial cells (Figure 6b). No signs of intracellular h-FTAA binding were observed.

\section{Intracellular cellulose detected by h-FTAA in S. Typhimurium infection}

Biofilm is considered an important factor in the pathogenesis of Salmonella infections. Shifting to the common model serovar S. Typhimurium, we first confirmed that its ECM components are also detectable by h-FTAA. Fluorescent spectral profiling of biofilm formed by the S. Typhimurium strain 14028 ssaG:: fp $^{+}$ (Supplementary Table 1) showed the same distinctive emission spectra as $S$. Enteritidis, with a signature cellulose peak at $480 \mathrm{~nm}$ (Supplementary Figure 6a). h-FTAA also proved useful for kinetic, real-time recordings of curli and cellulose production in growing cultures (Supplementary Figure 6b), and for microscopy-based visualisation of the mesh-like ECM in S. Typhimurium strain 14028 ssaG::gfp ${ }^{+}$(Figure 6c). Due to structural similarities, the cellulose polysaccharide can thus be successfully identified by h-FTAA across different S. Enterica serovars.
S. Typhimurium is a facultative intracellular pathogen that proliferates in phagocytic and non-phagocytic cells. ${ }^{29}$ We analysed whether h-FTAA could identify cellulose produced during the intracellular life cycle of 14028 ssaG:: gfp ${ }^{+}$. Infected epithelial cells and macrophages were incubated in h-FTAA, then analysed under confocal microscopy. Using optical settings optimised for cellulose detection $\left(\lambda_{\mathrm{Ex}} 473 \mathrm{~nm}, \lambda_{\mathrm{Em}} 550-655 \mathrm{~nm}\right.$ ), red fluorescence was observed that co-localised to GFP-expressing bacteria (Figure $6 \mathrm{~d}, \mathrm{e}$, Supplementary Movies 1 and 2). These data were extended to tissue infection, where cellulose stained by h-FTAA was observed in association with bacteria inside the liver of infected mice (Figure 6f). Collectively, our data demonstrate biofilm formation as an integrated feature of Salmonella's intracellular lifestyle, and corroborates recent findings demonstrating cellulose production by Salmonella inside macrophages. ${ }^{30}$

\section{DISCUSSION}

The essential role of ECM in establishing sessile and planktonic biofilms is well recognised. ${ }^{31,32}$ Expression of ECM is dynamically and environmentally regulated, ${ }^{33,34}$ highlighting the importance of monitoring production of these components in real-time in the endogenous environment. The current lack of methods for direct labelling and discrimination of ECM components under live conditions has severely hampered our understanding of these processes. The biofilm optotracing technology described here is designed to overcome this shortage. A major advantage is that this method enables direct tracing of ECM production in situ under a variety of conditions, including liquid cultures and growth on biotic and abiotic surfaces. Acting as structure-responsive chameleons, altered geometry of the highly photostable LCO molecules, due to the structural orientation of the thiophene backbone when bound to a target, is instantly translated into altered intensity and spectral properties of emitted light at given wavelengths. When applied as a non-toxic medium additive, LCOs continuously incorporates into the growing biofilm, emitting target-specific opto-signals immediately as the target is produced. This circumvents the conventional problem of dye penetration into ECM and enables true real-time analysis. By eliminating fixation and washing procedures, the one-step biofilm optotracing technology is ideal for studies of weakly attached and pellicle biofilms. In comparison with the gold standard CV assay that is limited to end-point experiments, our method was shown to be dynamically and molecularly superior, offering simultaneous tracing and differentiation of ECM production in real-time. We demonstrate here the biofilm optotracing technology using two Salmonella serovars, the method should be widely applicable to any bacterial species producing amyloid curli protein and cellulose polysaccharide.

Conceptually, the optotracing technology differs not only from traditional dyes, but also from antibody labelling. Whereas specificity of the latter lies in the primary antibody-ligand interaction, the binding pattern of LCO molecules shows a degree of promiscuity. Signal specificity is rather obtained by features such as the exclusive combination of excitation and emission wavelengths for explicit combinations of sensor and target molecules, and the inherent ON/OFF switching that produces markedly increased emission only when target proteins and polysaccharides are detected. The optotracing technology thus represents a one-step process, which eliminates the need of fluorophore-conjugated antibodies or lectins for detection.

We see the reported technology as complementary to current methods in biofilm research. In imaging applications, the optotracing technology helps to overcome the shortage of polysaccharide-targeting antibodies, and supplements the use of lectins. In molecular studies, combining the dynamic optotracing technology with transcriptomic and proteomic studies of end-stage bacterial cultures will promote better understanding of 
the dynamic regulatory systems governing biofilm formation. One example is curli expression, reported to occur during stationary phase $^{33-35}$ as its biosynthesis depends on the biofilm regulator CsgD, whose expression requires the stationary-phase sigma factor RpoS. ${ }^{3,36}$ Parallel measurements of turbidity and the presence of curli and cellulose during exponential growth in batch culture imply, however, that transcription and translation of ECM factors occur already in mid-exponential phase, since assembled curli fibres and cellulose polysaccharides are detected extracellularly at late exponential/early stationary phase. Dynamic recordings also suggest that compensatory mechanisms modulate biofilm formation, demonstrated by cellulose expression occurring ca $4 \mathrm{~h}$ earlier in a curli-negative strain compared to the wt. The molecular mechanisms governing co-regulation of curli and cellulose remains to be elucidated. The biofilm optotracing technology is also well suited for analysing bacterial colonies on solid media. Whereas traditional rdar morphotyping is visually determined on CR- or calcofluor-containing agar plates, LCOs provide an unbiased method for specific determination of ECM components as soon as colonies appear on the LB agar plates. The timesaving aspect is further enhanced, since screening for the biofilm phenotype on LB agar allows colonies originating from a single bacterium to be used immediately for further genetic processing.

As with most technologies in their infancy, the method does have limitations. With the limited set of LCOs and target molecules tested to date, the frequency of target molecules adopting similar geometry, thereby producing overlapping optical spectra, is currently unknown. Also, the presence of unknown molecules in complex media also poses a risk of unexpected binding. This was illustrated when h-HTAA was used to monitor biofilm formation in unwashed cultures, where a myriad of molecules obscured the signals from curli and cellulose. However, this shortcoming was not observed for the chemically related $h$-FTAA, verifying that a minor chemical modification of the LCO, e.g., varying the spacing between the carboxyl groups along thiophene backbone, can improve the performance of the LCO. Recent studies have also shown that specificity towards distinct biomolecular targets can be achieved by adding proper $\beta$ side chain functionalities along the thiophene backbone and that replacement of the central thiophene moiety with other heterocyclic moieties can generate a palette of LCOs with distinct emission profiles covering the visual spectrum. ${ }^{37,38}$ Overall, we foresee that similar chemical modifications of the LCOs will be extremely useful for overpowering potential limitations of the method.

The molecular sensors used in this study belong to the exciting group of electroactive polymers, whose electronic and ionic conductivity only recently has started to be explored in infection research. ${ }^{39}$ The biofilm optotracing technology presented here represents the first photophysical application of conducting polymers in infection research, thereby widening the opportunities of organic conducting polymers in infection biology. Ongoing work in our group is aimed at defining further LCOs binding characteristics to different elements of the ECM as well as across a wide-range of bacterial species.

\section{METHODS}

h-HTAA and h-FTAA

The luminescent conjugated oligothiophene h-HTAA and h-FTAA were synthesised as previously described ${ }^{16,17,40}$ according to the scheme in Supplementary Figure 7 . Stock solutions $(1.5 \mathrm{mmol} / \mathrm{l})$ prepared in ${ }^{\mathrm{D}_{2} \mathrm{O}}$ were maintained in the fridge. Both LCOs were used at $3 \mu \mathrm{mol} / \mathrm{l}$ final concentration, which is predicted to be in excess based on the original article. ${ }^{16}$

Bacterial strains, media, generation time and viability assay Bacterial strains (Supplementary Table 1) were routinely cultured on Luria-Bertani (LB) agar or in LB broth at $37^{\circ} \mathrm{C}$. LB without (w/o) salt was used to promote biofilm formation at $28^{\circ} \mathrm{C}$. The GFP-encoding plasmid p2777, purified from S. Typhimurium NCTC 12023 by QIAprep Spin kit
(Qiagen, Hilden, Germany), was passaged via transfer strain ARD83 into S. Enteritidis $3934 \mathrm{wt}$ and isogenic strains by electroporation. ${ }^{41}$ Positive clones were selected on LB plates with ampicillin $(100 \mu \mathrm{g} / \mathrm{ml})$. Generation time was assessed by diluting cultures of $S$. Enteritidis 3934 at $\mathrm{OD}_{600}=0.6$, to $10^{5} \mathrm{CFU} / \mathrm{ml}$ in LB. Following transfer of $70 \mathrm{ml}$ to separate flasks, $210 \mu \mathrm{l}$ of the stock solutions of h-FTAA, and h-HTAA or of PBS was added. OD $_{600}$ of cultures incubated at $37^{\circ} \mathrm{C}, 230$ r.p.m., was recorded every $10 \mathrm{~min}$ to define generation time. Viable count was obtained by spread plating $100 \mu \mathrm{l}$ withdrawn from the culture at indicated times onto LB agar plates.

\section{Congo red assay}

Morphotypes based on curli and cellulose expression were observed after growth at $28{ }^{\circ} \mathrm{C}$ for $1-3$ days on LB plates w/o salt, supplemented with Congo red $(40 \mu \mathrm{g} / \mathrm{ml}$, Sigma-Aldrich, Stockholm, Sweden) and Coomassie brilliant blue G-250 (20 $\mu \mathrm{g} / \mathrm{ml}$, Sigma) dissolved in $70 \%$ ethanol (Kemetyl $A B$, Haninge, Sweden).

\section{Inclined coverslip assay}

Exponentially growing cultures $\left(37^{\circ} \mathrm{C}, 230\right.$ r.p.m.) of indicated strains were harvested at $\mathrm{OD}_{600}=0.6$, diluted to $10^{5} \mathrm{CFU} / \mathrm{ml}$ in $\mathrm{LB}$ w/o salt, dispensed (8 $\mathrm{ml}$ aliquots) in six-well plates (Sarstedt, Numbrecht, Germany) with inclined glass coverslips $(24 \times 24 \mathrm{~mm}$, VWR International, Stockholm, Sweden), and incubated for $48 \mathrm{~h}$ at $28^{\circ} \mathrm{C}$ to allow biofilm formation. Removed coverslips were washed twice in PBS before and after $1 \mathrm{~h}$ fixation in $4 \%$ formaldehyde, then immersed in $3 \mu \mathrm{mol} / \mathrm{l}$ h-HTAA or h-FTAA in PBS. Following $30 \mathrm{~min}$ incubation in the dark, coverslips were washed twice in PBS, then prepared for microscopy analysis. In negative controls, coverslips were immersed in PBS without added LCOs. Biofilm formed on the surface at the air-liquid interface was analysed on coverslips immobilised (Vectashield, VWR International) onto glass slides. The $20 \times$ dry objective on an Olympus FV1000 confocal microscope (Olympus, Stockholm, Sweden) was used with preset detection settings for transmission, GFP (h-HTAA) and Cy3 (h-FTAA) fluorescence.

\section{Small-volume 96-well biofilm assays}

Semi-high-throughput biofilm recordings were achieved by downsizing the conventional 24-well plate format to the scale of a 96-well plate. Bacterial overnight cultures $\left(37^{\circ} \mathrm{C}, 230\right.$ r.p.m.) were diluted in LB w/o salt to $10^{5} \mathrm{CFU} / \mathrm{ml}$. Aliquots were made to which $\mathrm{h}$-HTAA or $\mathrm{h}$-FTAA ( $3 \mu \mathrm{mol} / \mathrm{l}$ final concentrations), or PBS was added. Similar mixtures without bacteria were used as negative controls. $50 \mu \mathrm{l}$ of each mixture, dispensed in triplicates into tissue culture treated 96-well plates (Sarstedt, Hilden, Germany) were incubated at $28^{\circ} \mathrm{C}$. To prevent desiccation, sterile water (300 $\mathrm{\mu l}$ ) was added to each unused well and to fill the entire inter-well region (Supplementary Figure 2). At selected time points, supernatants were discarded and wells were rinsed twice with $150 \mu \mathrm{l}$ PBS prior to analysis.

Conventional biofilm analysis was performed by adding $100 \mu \mathrm{l}, 0.4 \%$ Crystal violet (CV) (Sigma-Aldrich) to wells with PBS originally added to the culture. After $10 \mathrm{~min}$ in room temperature, wells were rinsed twice with PBS. Surface-attached CV was released following incubation of wells in $125 \mu \mathrm{l} 99.5 \% \mathrm{EtOH}$ for $15 \mathrm{~min}$ at room temperature. $100 \mu \mathrm{l}$ from each well was transferred to a new plate and the CV absorbance was recorded at $540 \mathrm{~nm}$ in a Synergy Mx Monochromator-Based Multi-Mode Microplate Reader (Biotek, Bad Friedrichshall, Germany).

In parallel, biofilm was quantified in wells with cultures originally supplemented with h-HTAA and h-FTAA. Direct comparison of biofilm amounts in this and the CV assay was achieved by exposing LCO-supplemented wells to exactly the same experimental procedures, using PBS instead of $\mathrm{CV}$ and $\mathrm{EtOH}$. In the final steps, $100 \mu \mathrm{l}$ from each well was transferred into a new plate, and optical recording of LCOs was achieved by excitation at $405 \mathrm{~nm}$ and emission detected at $545 \mathrm{~nm}$ (h-HTAA) and $556 \mathrm{~nm}$ (h-FTAA) using the plate reader.

\section{LCO-based biofilm analysis under non-disruptive conditions}

Bacterial cultures supplemented with h-HTAA and h-FTAA ( $3 \mu \mathrm{mol} / \mathrm{l}$ final concentration) were seeded into the small-volume 96 -well biofilm assay as described. Non-disruptive biofilm recording was achieved by positioning the seeded plate in a Synergy Mx Monochromator-Based Multi-Mode Microplate Reader (Biotek), and recording excitation spectra of the growing culture at indicated times. Excitation spectra were collected at $300-500 \mathrm{~nm}$, with emission detected at $545 \mathrm{~nm}$. Curli-specific emission spectra were collected by exciting cultures at $405 \mathrm{~nm}$ and reading 
emission at 500-700 nm. Cellulose-specific emission spectra were collected by excitation at $500 \mathrm{~nm}$ and emission recorded at $520-700 \mathrm{~nm}$.

\section{LCO-based cellulose detection}

Serial two-fold dilutions, ranging from $5-0.04 \mathrm{mg} / \mathrm{ml}$, of microcrystalline

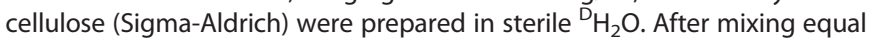
volumes of cellulose suspensions and h-FTAA ( $3 \mu \mathrm{mol} / \mathrm{l}$ final concentration), $50 \mu \mathrm{l}$ of each mix was transferred to a 96-well plate, which was placed in a Synergy Mx Monochromator-Based Multi-Mode Microplate Reader (Biotek). Excitation spectra in the range 300-500 nm were collected with emission detected at $545 \mathrm{~nm}$.

\section{Morphotyping of biofilms}

The conventional method for Congo red-based rdar morphotyping ${ }^{42,43}$ was adapted to enable LCO-based spectral morphotyping of biofilms. Cultures (LB medium, $37^{\circ} \mathrm{C}, 230$ r.p.m.) of the 3934 wt strain, and isogenic mutant strains $\triangle b c s A, \triangle \operatorname{csg} A$, and $\Delta \operatorname{csg} D$ harvested in exponential phase $\left(\mathrm{OD}_{600}=0.6\right)$ were diluted by a factor of $10^{7}$ in PBS. A volume of $25 \mu \mathrm{l}$ of each dilution was spotted on Congo red assay plates ${ }^{42}$ and LB agar plates w/o salt, and incubated at $28^{\circ} \mathrm{C}$ for $48 \mathrm{~h}$. The rdar morphology was documented on the Congo red plates. For LCO-based spectral morphotyping, single colonies from the LB plates were harvested with a loop and dissolved in $500 \mu \mathrm{l}$ PBS. After four cycles of $5 \mathrm{~s}$ sonication and $10 \mathrm{~s}$ pause, $25 \mu \mathrm{l}$ from each suspension was mixed with $25 \mu \mathrm{l}$ of $6 \mu \mathrm{mol} / \mathrm{l}$ h-FTAA and transferred to a 96-well plate. As control, h-FTAA added to PBS or to a cellulose (Sigma-Aldrich) suspension $(0.1 \mathrm{mg} / \mathrm{ml}$ in PBS) was used. Excitation spectra in the range $300-500 \mathrm{~nm}$ were collected with emission detected at $545 \mathrm{~nm}$.

To monitor the kinetics of ECM production, $100 \mu \mathrm{l}$ from an exponential $\left(\mathrm{OD}_{600}=0.6\right)$ culture of $3934 \mathrm{wt}$ was diluted by a factor of $10^{5}$, then spread plated on Congo red assay plates ${ }^{42}$ and LB agar plates w/o salt, and incubated at $28^{\circ} \mathrm{C}$. At days 1,2 and 3 , the rdar morphology of single-cell colonies on Congo red plates was documented. In parallel, single-cell colonies on LB plates were harvested with a loop and dissolved in $500 \mu \mathrm{l}$ PBS. After four cycles of $5 \mathrm{~s}$ sonication and $10 \mathrm{~s}$ pause, $25 \mu \mathrm{l}$ from each suspension was mixed with $25 \mu \mathrm{l}$ of $6 \mu \mathrm{mol} / \mathrm{l} \mathrm{h}$-FTAA and transferred to a 96-well plate. As control, h-FTAA added to PBS or to a cellulose (SigmaAldrich) suspension $(0.1 \mathrm{mg} / \mathrm{ml}$ in PBS) was used. Excitation spectra in the range $300-500 \mathrm{~nm}$ were collected with emission detected at $545 \mathrm{~nm}$.

\section{Real-time kinetics of ECM expression during bacterial growth in} liquid cultures

Cultures $\left(10^{5} \mathrm{CFU} / \mathrm{ml}\right)$ of strain $3934 \mathrm{wt}$, and isogenic mutants $\triangle b c s A$-, $\Delta \operatorname{csg} A$ - and $\Delta \operatorname{csg} D$-containing plasmid p2777 were prepared in LB w/o salt from an overnight culture. ${ }^{44}$ After addition of h-FTAA $(3 \mu \mathrm{mol} / \mathrm{l}$ final concentration), $50 \mu \mathrm{l}$ aliquots were seeded into four parallel 96 -well plates, which were placed in a $28^{\circ} \mathrm{C}$ incubator. Every hour, one plate was positioned in a Synergy Mx Monochromator-Based Multi-Mode Microplate Reader (Biotek), allowing $4 \mathrm{~h}$ intervals between scans of individual plates to minimise culturing disturbances. Bacterial culture density was monitored by GFP fluorescence intensity $\left(\lambda_{\mathrm{Ex}} 445 \mathrm{~nm}, \lambda_{\mathrm{Em}} 510 \mathrm{~nm}\right)$ for a period of $48 \mathrm{~h}$. Simultaneously, curli $\left(\lambda_{\mathrm{Ex}} 405 \mathrm{~nm}, \lambda_{\mathrm{Em}} 556 \mathrm{~nm}\right)$ and cellulose $\left(\lambda_{\mathrm{Ex}} 500 \mathrm{~nm}\right.$, $\lambda_{\mathrm{Em}} 600 \mathrm{~nm}$ ) were recorded. A time series was obtained by combining readings from the four plates. Relative fluorescence units of GFP and of h-FTAA binding to curli and cellulose are presented in the same graphs to visualise the hourly change in signals.

Live fluorescence confocal imaging of h-FTAA-stained biofilm ECM h-FTAA ( $3 \mu \mathrm{mol} / \mathrm{l}$ final concentration) was added to cultures $\left(10^{5} \mathrm{CFU} / \mathrm{ml}\right.$ ) of strain 3934 wt p2777 and S. Typhimurium strain 14028 ssaG.::gfp ${ }^{+}$ prepared in LB w/o salt. A volume of $8 \mathrm{ml}$ aliquots were transferred into six-well plates with inclined glass coverslips to allow biofilm formation at the air-liquid interface during $48 \mathrm{~h}$ incubation in $28^{\circ} \mathrm{C}$. Biofilm formed on the removed coverslips, washed twice with PBS, were analysed by fluorescence confocal microscopy (FV1000 confocal microscope, Olympus) using a UPLSAPO $40 \times 2$ (NA 0.95) lens and a UPLSAPO $60 \times W$ (NA 1.2) water immersion lens (Olympus). In parallel, control experiments were performed using bacteria cultured in the absence of h-FTAA, or h-FTAA added to strain 3934 wt lacking the GFP expressing plasmid.
Intracellular cellulose expression stained by h-FTAA

Macrophage-like cell line RAW264.7 (TIB-71; ATCC, Manassas, VA, USA) was propagated in supplemented RPMI 1640 (Gibco, Paisley, UK) ${ }^{45}$ and the epithelial cell line CRL-4031 (ATCC) in supplemented DMEM/F12 (Gibco, Paisley, UK) ${ }^{46}$ as described. S. Typhimurium strain 14028 ssaG:: gfp ${ }^{+}$grown in $\mathrm{LB}, 37^{\circ} \mathrm{C}$, was harvested at $\mathrm{OD}_{600}=0.6$, resuspended in respective cell culture media, and used at multiplicity of infection $(\mathrm{MOI})=10$ to infect RAW264.7 and CRL-4031 cells seeded on round coverslips in 24-well plates. Media were exchanged after $90 \mathrm{~min}$ to gentamicin-containing $(50 \mu \mathrm{g} / \mathrm{ml}$, Sigma-Aldrich) media, and incubation proceeded for $30 \mathrm{~min}$ to kill extracellular bacteria. After a shift to maintenance medium $(10 \mu \mathrm{g} / \mathrm{ml}$ gentamicin) with infection proceeding for $90 \mathrm{~min}$, supernatants were discarded. Cells were rinsed twice in PBS, fixed in $4 \%$ paraformaldehyde for $1 \mathrm{~h}$, and rinsed twice in PBS. Fixed cells were then treated with ice-cold acetone for $4 \mathrm{~min}$, rinsed twice in PBS and stained with Hoechst 33342 (Life Technologies, Stockholm, Sweden), Alexa Fluor 647 Phalloidin (Life Technologies) and h-FTAA ( $3 \mu \mathrm{mol} / \mathrm{l})$ in PBS for $1 \mathrm{~h}$. Coverslips washed twice in PBS were mounted on microscope slides using Prolong Gold (Life Technologies). Fluorescence confocal microscopy (FV1000 confocal microscope (Olympus), using a UPLSAPO $60 \times$ W (NA 1.2) water immersion lens (Olympus) was performed using optical settings for Hoechst 33342, GFP ${ }^{+}\left(\lambda_{\mathrm{Ex}} 473 \mathrm{~nm}, \lambda_{\mathrm{Em}} 490-540 \mathrm{~nm}\right)$ and h-FTAA $\left(\lambda_{\mathrm{Ex}}\right.$ $473 \mathrm{~nm}, \lambda_{\mathrm{Em}} 590-655 \mathrm{~nm}$ ). The imaging processing software Fiji (ImageJ, Bethesda, MD, USA) was used for image analysis. ${ }^{47}$ Three-dimensional projections were generated by brightest point projection of image stacks ( $10 \mu \mathrm{m}$ stack of $0.5 \mu \mathrm{m}$ steps) and processed into.MOV files of $15 \mathrm{fps}$.

Mice (6-8 weeks BALB/c, Taconic Europe, Lille Skensved, Denmark) were intraperitoneally injected with $10^{4}$ CFU S. Typhimurium strain 14028 ssaG:: $\mathrm{gfp}^{+48}$ in $100 \mu \mathrm{l} \mathrm{PBS}$, and the control group with PBS only. Livers excised from mice 3 days post infection were cut in $5 \mathrm{~mm}$ pieces that were immediately snap-frozen in a slurry of dry ice $/ 70 \%$ ethanol. Ten micrometre sections were treated with ice-cold acetone for $4 \mathrm{~min}$ and rinsed twice in PBS. Sections were stained with Hoechst 33342, h-FTAA $(3 \mu \mathrm{mol} / \mathrm{l})$ in PBS and/or Alexa Fluor 647 Phalloidin (Life Technologies) for $1 \mathrm{~h}$, washed twice with PBS, and mounted in Prolong Gold (Life Technologies). Fluorescence confocal microscopy (FV1000 confocal microscope, Olympus) was performed using the same optical settings as for infected cell lines. Mice were housed at MTC animal facility (Karolinska Institutet, Stockholm, Sweden) in accordance with institutional and national guidelines (ethical permit N491/11, Stockholm Norra Djurförsöksetiska Nämnd).

\section{Statistical analysis}

All data were organised and processed with GraphPad Prism 6 (Graphpad Software, La Jolla, CA, USA). To determine the significance of the observed difference in cellulose expression kinetics, cellulose-specific relative fluorescence units collected from growing biofilm cultures of strain 3934 $w t+P 2777$ and $\Delta c s g A+P 2777$ were first normalised to eliminate differences in intensities such that the strongest and weakest relative fluorescence units was represented by $100 \%$ and $0 \%$, respectively. Significance analysis was then performed by two-way Anunivariate analysis of variance (ANOVA) with multiple comparisons, in which the mean of cells from each row from each strain is compared.

\section{ACKNOWLEDGEMENTS}

We thank Professor P Hammarström (Linköping University) for careful review of the manuscript. We thank the Erling-Perssons Family Foundation, the Swedish Foundation for Strategic Research, the Swedish Research Council-Medicine and Carl Bennet AB for financial support, and acknowledge the Knut and Alice Wallenberg Foundation (CLICK facility).

\section{CONTRIBUTIONS}

FXC, KPRN and ARD designed research; MB and LBGJ performed chemical synthesis; FXC, SF, KM and MR performed bacterial research; FXC, MR, KPRN and ARD analysed data; FXC, KPRN and ARD wrote the manuscript with input from KM and MR.

\section{COMPETING INTERESTS}

SF, LBGJ, KM and MR declare no competing financial interest. FXC and ARD are co-inventors on a pending patent. FXC, MB, KPRN and ARD are part owners of a company, which may commercialise LCOs for uses as described in this article. 


\section{REFERENCES}

1. Hall-Stoodley, L., Costerton, J. W. \& Stoodley, P. Bacterial biofilms: from the natural environment to infectious diseases. Nat. Rev. Microbiol. 2, 95-108 (2004).

2. Hood, S. K. \& Zottola, E. A. Adherence to stainless steel by foodborne microorganisms during growth in model food systems. Int. J. Food Microbiol. 37, 145-153 (1997).

3. Römling, U., Sierralta, W. D., Eriksson, K. \& Normark, S. Multicellular and aggregative behaviour of Salmonella typhimurium strains is controlled by mutations in the agfD promoter. Mol. Microbiol. 28, 249-264 (1998).

4. Prouty, A. M. \& Gunn, J. S. Comparative analysis of Salmonella enterica serovar Typhimurium biofilm formation on gallstones and on glass. Infect. Immun. 71, 7154-7158 (2003).

5. Ngwai, Y. B., Adachi, Y., Ogawa, Y. \& Hara, H. Characterization of biofilm-forming abilities of antibiotic-resistant Salmonella typhimurium DT104 on hydrophobic abiotic surfaces. J. Microbiol. Immunol. Infect. 39, 278-291 (2006).

6. Ledeboer, N. A. \& Jones, B. D. Exopolysaccharide sugars contribute to biofilm formation by Salmonella enterica serovar typhimurium on HEp-2 cells and chicken intestinal epithelium. J. Bacteriol. 187, 3214-3226 (2005).

7. Ledeboer, N. A., Frye, J. G., McClelland, M. \& Jones, B. D. Salmonella enterica serovar Typhimurium requires the Lpf, Pef, and Tafi fimbriae for biofilm formation on HEp-2 tissue culture cells and chicken intestinal epithelium. Infect. Immun. 74, 3156-3169 (2006).

8. Sutherland, I. W. The biofilm matrix--an immobilized but dynamic microbial environment. Trends Microbiol. 9, 222-227 (2001).

9. Flemming, H. C. \& Wingender, J. The biofilm matrix. Nat. Rev. Microbiol. 8, 623-633 (2010).

10. Solano, C. et al. Genetic analysis of Salmonella enteritidis biofilm formation: critical role of cellulose. Mol. Microbiol. 43, 793-808 (2002).

11. Costerton, J. W., Lewandowski, Z., Caldwell, D. E., Korber, D. R. \& Lappin-Scott, H. M. Microbial biofilms. Annu. Rev. Microbiol. 49, 711-745 (1995).

12. Pedersen, K. Method for studying microbial biofilms in flowing-water systems. Appl. Environ. Microbiol. 43, 6-13 (1982).

13. Groenning, M. Binding mode of Thioflavin $T$ and other molecular probes in the context of amyloid fibrils-current status. J. Chem. Biol. 3, 1-18 (2010).

14. Klunk, W. E., Pettegrew, J. W. \& Abraham, D. J. Quantitative evaluation of congo red binding to amyloid-like proteins with a beta-pleated sheet conformation. J. Histochem. Cytochem. 37, 1273-1281 (1989).

15. Klingstedt, T. et al. The structural basis for optimal performance of oligothiophene-based fluorescent amyloid ligands: conformational flexibility is essential for spectral assignment of a diversity of protein aggregates. Chemistry 19, 10179-10192 (2013).

16. Klingstedt, T. et al. Synthesis of a library of oligothiophenes and their utilization as fluorescent ligands for spectral assignment of protein aggregates. Org. Biomol. Chem. 9, 8356-8370 (2011).

17. Aslund, A. et al. Novel pentameric thiophene derivatives for in vitro and in vivo optical imaging of a plethora of protein aggregates in cerebral amyloidoses. ACS Chem. Biol. 4, 673-684 (2009).

18. Heilbronner, G. et al. Seeded strain-like transmission of $\beta$-amyloid morphotypes in APP transgenic mice. EMBO Rep. 14, 1017-1022 (2013).

19. Berg, I., Nilsson, K. P. R., Thor, S. \& Hammarström, P. Efficient imaging of amyloid deposits in Drosophila models of human amyloidoses. Nat. Protoc. 5, 935-944 (2010).

20. Mahajan, V. et al. Cross $\beta$-sheet conformation of keratin 8 is a specific feature of Mallory-Denk bodies compared with other hepatocyte inclusions. Gastroenterology 141, 1080-1090.e1 (2011).

21. Sigurdson, C. J. et al. Prion strain discrimination using luminescent conjugated polymers. Nat. Methods 4, 1023-1030 (2007).

22. Latasa, C. et al. BapA, a large secreted protein required for biofilm formation and host colonization of Salmonella enterica serovar Enteritidis. Mol. Microbiol. 58 1322-1339 (2005).

23. Herrmann, U. S. et al. Structure-based drug design identifies polythiophenes as antiprion compounds. Sci. Transl. Med. 7, 123 (2015).

24. Brelstaff, J. et al. The fluorescent pentameric oligothiophene pFTAA identifies filamentous tau in live neurons cultured from adult P301S tau mice. Front. Neurosci. 9, 184 (2015).

25. Römling, U. Molecular biology of cellulose production in bacteria. Res. Microbiol. 153, 205-212 (2002).

26. Serra, D. O., Richter, A. M. \& Hengge, R. Cellulose as an architectural element in spatially structured Escherichia coli biofilms. J. Bacteriol. 195, 5540-5554 (2013).

27. Römling, U., Bian, Z., Hammar, M., Sierralta, W. D. \& Normark, S. Curli fibers are highly conserved between Salmonella typhimurium and Escherichia coli with respect to operon structure and regulation. J. Bacteriol. 180, 722-731 (1998).

28. Gabrielsson, E. O., Tybrandt, K., Hammarström, P., Berggren, M. \& Nilsson, K. P. R. Spatially controlled amyloid reactions using organic electronics. Small 6, 2153-2161 (2010).

29. de Jong, H. K., Parry, C. M., van der Poll, T. \& Wiersinga, W. J. Host-pathogen interaction in invasive Salmonellosis. PLoS Pathog. 8, e1002933 (2012).

30. Pontes, M. H., Lee, E. J., Choi, J. \& Groisman, E. A. Salmonella promotes virulence by repressing cellulose production. Proc. Natl. Acad. Sci. USA 112, 5183-5188 (2015).

31. Hobley, L., Harkins, C., MacPhee, C. E. \& Stanley-Wall, N. R. Giving structure to the biofilm matrix: an overview of individual strategies and emerging common themes. FEMS Microbiol. Rev. 39, 649-669 (2015).

32. Peterson, B. W. et al. Viscoelasticity of biofilms and their recalcitrance to mechanical and chemical challenges. FEMS Microbiol. Rev. 39, 234-245 (2015).

33. Brombacher, E., Dorel, C., Zehnder, A. J. B. \& Landini, P. The curli biosynthesis regulator CsgD co-ordinates the expression of both positive and negative determinants for biofilm formation in Escherichia coli. Microbiology. 149, 2847-2857 (2003).

34. Mika, F. et al. Targeting of csgD by the small regulatory RNA RprA links stationary phase, biofilm formation and cell envelope stress in Escherichia coli. Mol. Microbiol. 84, 51-65 (2012).

35. Van Gerven, N., Klein, R. D., Hultgren, S. J. \& Remaut, H. Bacterial amyloid formation: structural insights into curli biogensis. Trends Microbiol. 23 693-706 (2015).

36. Römling, U., Rohde, M., Olsén, A., Normark, S. \& Reinköster, J. AgfD, the checkpoint of multicellular and aggregative behaviour in Salmonella typhimurium regulates at least two independent pathways. Mol. Microbiol. 36, 10-23 (2000)

37. Shirani, H. et al. A palette of fluorescent thiophene-based ligands for the identification of protein aggregates. Chemistry 21, 15133-15137 (2015).

38. Cieślar-Pobuda, A. et al. Cell type related differences in staining with pentameric thiophene derivatives. Cytometry A 85, 628-635 (2014).

39. Löffler, S., Libberton, B. \& Richter-Dahlfors, A. Organic bioelectronics in infections J. Mater. Chem. B 3, 4979-4992 (2015).

40. Aslund, A. et al. Studies of luminescent conjugated polythiophene derivatives: enhanced spectral discrimination of protein conformational states. Bioconjug. Chem. 18, 1860-1868 (2007).

41. Bullas, L. R. \& Ryu, J. I. Salmonella typhimurium LT2 strains which are $r-m+$ for all three chromosomally located systems of DNA restriction and modification. J. Bacteriol. 156, 471-474 (1983).

42. Römling, U. Characterization of the rdar morphotype, a multicellular behaviour in Enterobacteriaceae. Cell Mol. Life Sci. 62, 1234-1246 (2005).

43. Bokranz, W., Wang, X., Tschäpe, H. \& Römling, U. Expression of cellulose and curli fimbriae by Escherichia coli isolated from the gastrointestinal tract. J. Med. Microbiol. 54, 1171-1182 (2005).

44. Hansen-Wester, I., Chakravortty, D. \& Hensel, M. Functional transfer of Salmonella pathogenicity island 2 to Salmonella bongori and Escherichia coli. Infect. Immun. 72, 2879-2888 (2004).

45. Raschke, W. C., Baird, S., Ralph, P. \& Nakoinz, I. Functional macrophage cell lines transformed by Abelson leukemia virus. Cell 15, 261-267 (1978).

46. Wieser, M. et al. hTERT alone immortalizes epithelial cells of renal proximal tubules without changing their functional characteristics. Am. J. Physiol. Renal Physiol. 295, F1365-F1375 (2008).

47. Schindelin, J. et al. Fiji: an open-source platform for biological-image analysis. Nat. Methods 9, 676-682 (2012).

48. Hautefort, I., Proença, M. J. \& Hinton, J. C. D. Single-copy green fluorescent protein gene fusions allow accurate measurement of Salmonella gene expression in vitro and during infection of mammalian cells. Appl. Environ. Microbiol. 69, 7480-7491 (2003).

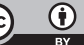

This work is licensed under a Creative Commons Attribution 4.0 International License. The images or other third party material in this article are included in the article's Creative Commons license, unless indicated otherwise in the credit line; if the material is not included under the Creative Commons license, users will need to obtain permission from the license holder to reproduce the material. To view a copy of this license, visit http://creativecommons.org/licenses/ by/4.0/

(c) The Author(s) 2016

Supplementary Information accompanies the paper on the npj Biofilms and Microbiomes website (http://www.nature.com/npjbiofilms) 